\title{
Phacomatoses, genetic testing for personalisation of clinical management (part 1.)
}

\author{
Anna Kofla-Dłubacz ${ }^{1}$, Andrzej Stawarski ${ }^{1}$, Tomasz Pytrus ${ }^{1}$, Justyna Gil² \\ $12^{\text {nd }}$ Department of Paediatrics, Gastroenterology and Nutrition, Faculty of Medicine, Wroclaw Medical University, Wroclaw, Poland \\ ${ }^{2}$ Department of Genetics, Faculty of Medicine, Wroclaw Medical University, Wroclaw, Poland
}

Genetically determined disorders of tissue development, which are derived from the ecto-, endo- and mesoderm and develop in the early stages of foetal life, referred to as phacomatoses, constitute a large group of diseases predisposing to development of neoplasms. Early diagnosis, including identification of mutations and clinical evaluation, enables introduction of multidisciplinary care for patients with a confirmed diagnosis. Thus, the long-term prognosis and quality of patients' life can be improved. The most common phacomatoses include neurofibromatosis types 1 and 2 and schwannomatosis.

Key words: phacomatoses, neurocutaneous diseases, neurofibromatosis 1, neurofibromatosis 2

\section{Introduction}

Phacomatoses, also known as neurocutaneous diseases, constitute a heterogeneous group of genetically determined disorders of development of tissues derived from three germ layers: ecto-, endo- and mesoderm. They are manifested by skin, nervous and vascular lesions. Phacomatoses are associated with a significant increase in the risk of cancer [1]. Management of patients with a diagnosed phacomatosis involves multidisciplinary supervision with particular emphasis on the early detection of neoplastic changes. The most common phacomatoses include neurofibromatosis (neurofibromatosis types 1 and 2) and less frequent disorders, such as the following syndromes: Sturge-Weber, von Hippel-Lindau, ataxia-telangiectasia, Klippel-Trenaunay, GorlinGoltz, Schimmelpennin-Feuerstein-Mims, tuberous sclerosis and Osler-Weber-Rendu disease.

\section{Neurofibromatosis}

The historical classification (Carey et al.) of 1986 distinguished:

- type 1 neurofibromatosis (NF1),
- type 2 neurofibromatosis (NF2) - acoustic type,

- type 3 neurofibromatosis (NF3) - segmental type,

- type 4 neurofibromatosis (NF4) - familial type,

- type 5 neurofibromatosis (NF5) - the Noonan phenotype [2].

Nowadays, the neurofibromatosis group is defined to include neurofibromatosis type 1,2 and schwannomatosis, with neurofibromatosis type 1 accounting for $96 \%$ of all diagnoses [1].

\section{Neurofibromatosis type 1}

Type 1 neurofibromatosis (NF1, MIM \# 162200), historically known also as von Recklinghausen disease (after Friedrich Daniel von Reklinghausen, who described two cases of symptoms corresponding to NF1 in 1882), is an autosomal dominant condition with a prevalence of 1 in 3000 worldwide [2].

Clinical symptoms include skin, bone and vascular lesions, which display particular tendency to form benign and malignant tumours.

\section{How to cite:}

Kofla-Dłubacz A, Stawarski A, Pytrus T, Gil J. Phacomatoses, genetic testing for personalisation of clinical management (part 1.). NOWOTWORY J Oncol 2021 ; 71 :420-426. 
Pigmentation disorders are pathognomonic for NF1, but their severity may vary considerably between patients, even within the same family. Typical lesions include café au lait spots and excessive pigmentation of the armpits and groin (freckling in axillary and inguinal regions). Café au lait spots occur in 95-99\% of patients with NF1 [1]. They are often present at birth, and their number and size increase with age. Finding more than 6 spots of a diameter greater than $0.5 \mathrm{~cm}$ before puberty or greater than $1.5 \mathrm{~cm}$ after puberty is one of the diagnostic criteria for NF1 established at National Institute of Health (NIH) conference in 1988, which are still used as guidelines for the diagnosis of neurofibromatosis [3, 4]. Histopathological examination of pigmented lesions shows hyperpigmentation of the basal layer of the epidermis with macromelanosomes present [1]. These changes are not cancerous, but they constitute a visible cosmetic defect that reduces the patients' quality of life.

\section{Neurofibromas}

Neurofibromas occur in $60 \%$ of patients with NF1 [1]. These are benign tumours originating in the peripheral nerves' sheath, which may undergo malignant transformation. They proliferate from Schwann cells, epithelial cells, as well as from macrophages, mast cells, fibroblasts and pericytes and are divided into:

- cutaneous neurofibromas,

- subcutaneous/internal neurofibromas,

- plexiform neurofibromas.

Cutaneous neurofibromas appear as soft, sometimes pain-

ful and itchy growths that are skin-coloured or purple, can be unifocal or multiple, and cover a large part of the body's surface. Apart from the skin, neurofibromas may develop along the dorsal roots of the spinal cord or arise from nerve plexuses. By expanding and clustering (so-called plexiform neurofibromas - PN), they can compress adjacent anatomical structures and lead to neurological deficits, deformation of bone structures and structural and functional changes in internal organs.

They may transform into a tumour - malignant peripheral nerve sheath tumour (MPNST). The risk of MPNST in patients with NF1 is 1000 times higher in comparison to the general population $[5,6]$. The molecular basis of the transformation of plexiform nevi (PN) into MPNST has not been clearly explained, but it has been shown to be influenced by dysregulation of the RAS/MAPK signalling pathway (mitogen-activated protein kinase) and PI3K-AKT-mTOR [7, 8]. Schwann cells accumulated in the tumour mass are stimulated to malignant proliferation by growth factors secreted from the surrounding cells: mast cells, macrophages, and fibroblasts. A trauma - influencing the migration of mast cells - may stimulate neoplastic transformation [7].

The precancerous form of conversion of PN into MPNST is atypical neurofibroma (ANF) [5, 6]. The basic form of treatment for ANF and MPNTS is surgical resection. MPNST develops in $8-13 \%$ of patients and is the main cause of death in the course of NF1 [7, 9]. Surgical treatment with a large margin of the area around the tumour does not fully prevent local recurrence, which occurs in $25-37 \%$ of patients [10]. Pharmacological treatment of MPNST is ineffective. However, using doxorubicin, etoposide and ifosfamide in selected groups of patients may effectively reduce disease progression in metastatic and inoperable forms [11]. Inhibitors of the mTOR pathway, which are currently studied, seem to be promising [12]. The efficacy of other drugs used in clinical trials has not been demonstrated: tyrosine kinase inhibitors, erlotinib, sorafenib [13].

\section{Cancers}

Patients with NF1 have an increased risk of developing brain tumours compared to the general population. Brain tumours occurring in patients with NF1 are most often optic nerve gliomas (15-20\% of patients), gliomas of the brain stem, diencephalon and cerebellum [14].

Pilocytic astrocytoma (astrocytoma pilocyticum) is a lowgrade tumour (WHO I) which may undergo spontaneous remission in patients with NF1. During the time of the patient's development, so-called unidentified bright objects (UBO) may be observed, which are non-cancerous changes in the brain and dynamically arise and disappear. Their occurrence may influence the behavioural disorders of the ADHD type, mental retardation or epilepsy observed in patients [15].

Other cancers include:

- endocrine tumours, such as pheochromocytoma of the adrenal gland found in 5\% of patients with NF1 compared to below $1 \%$ in the general population [1]. Key clinical symptoms of phaeochromocytoma are arterial hypertension, arrhythmias, sudden flushing,

- gastrointestinal stromal tumours (GIST) occur in approximately 20\% of patients with NF1 [16]. They originate from the mesenchyme and arise from Cajal cells. Often small, long asymptomatic, they are detected during endoscopic and imaging examinations of the abdomen,

- endocrine tumours of the gastrointestinal tract that may occur in the course of NF1 include somatostatinoma and gastrinoma [16].

In childhood juvenile myelomonocytic leukemia (JMML), Wilms'tumour or rhabdomyosarcoma may also appear, as the risk is 20 times higher in patients with NF1 compared to the general population [1].

\section{Ocular symptoms}

Ocular symptoms include hamartomata-like Lisch nodules, which derive from pigment cells, fibroblasts and mast cells, presenting with characteristic irregularities of the iris in shades of brown [2]. These changes do not affect visual acuity and occur in over $90 \%$ of adult patients (the number increases with age). Optic gliomas (cancers of the anterior segment of the visual pathway) are an important manifestation of NF1. They are manifested by atrophy of the optic nerve and progressive loss of vision, as well as exophthalmia, nystagmus (described as 
see-haw nystagmus) and symptoms that result from increased intracranial pressure. Optic nerve gliomas occur in about 15\% of patients with NF1 and association of the tumour with NF1 accounts for $50 \%$ of all cases of this type of cancer [14]. Histologically, it is most often pilocytic astrocytoma. Its location limits the possibility of surgical intervention. Other ophthalmic symptoms found in NF1 include choroidal nevi and congenital glaucoma [17].

\section{Skeletal symptoms}

Skeletal symptoms include scoliosis, mainly of the cervico-thoracic region, pseudo-joints of long bones and bone dysplasia. Patients'height may be reduced due to bone deformities, and bone fractures are common. The mechanism of development of these changes has not been clarified, however, insufficient levels of vitamin $D_{3}$ and lower bone density compared to the general population were observed in patients with NF $[1,18]$.

\section{Cardiovascular symptoms}

Cardiovascular changes include, but are not limited to, narrowing of the pulmonary trunk, cerebral and renal arteries, which can lead to hypertension, myocardial infarction, and heart failure.

Treatment of neoplasms diagnosed in the course of type 1 neurofibromatosis is based on therapeutic protocols for forms unrelated to NF.

\section{Diagnostic criteria for neurofibromatosis type 1}

For diagnosis of type 1 neurofibromatosis, at least two of the following symptoms must be present [18]:

- six or more skin lesions of the café au lait type of a diameter greater than $5 \mathrm{~mm}$ in a child before puberty and greater than $15 \mathrm{~mm}$ after puberty,

- increased pigmentation of the armpits and groin,

- optic nerve glioma,

- two or more neurofibromas or one plexiform neurofibroma,

- first degree relative with neurofibromatosis type 1,

- two or more Lisch nodules (pigmented haemartomatous nodules of the retina) visible on a slit lamp examination,

- characteristic bone changes (dysplasia of the sphenoid bone, thinning of the cortex of long bones with or without pseudoarthrosis).

\section{Genetics}

Pathogenic variants of the NF1 gene constitute the molecular basis for the development of neurofibromatosis type 1. The NF1 gene is located on the long arm of chromosome 17 (locus q11.2) and encodes a 2818-aminoacid cytoplasmic protein, of 320kDa in mass, neurofibromin 1 (NF1, MIM* 613113) [19]. This gene contains 61 exons, 5 of which are subject to alternative splicing (9a, 10a-2, 23a, 43 and 48a), thus leading to the formation of different isoforms of neurofibromin. There are five isoforms of the protein known and their expression is characteristic for specific cells and / or tissues [20]. Neurofibromin is a multifunctional protein involved in numerous signalling pathways which regulates a number of cellular processes and plays a significant role in embryogenesis [21]. The presence of neurofibromin is detected in most cells of the body, but its highest expression is observed in cells of the nervous system such as astrocytes, oligodendrocytes and Schwann cells [20].

The main function of neurofibromin is to negatively regulate the Ras pathway by negative feedback through the activation of GTPase (GTPase activating protein - GAP). GAP regulation involves acceleration of the hydrolysis of GTP (guanosine-5'-triphosphate) associated with Ras to GDP (guanosine-5'-diphosphate) - by increasing the intrinsic activity of Ras GTApase. This "switches" the Ras oncogene to its inactive form [19]. Ras pathway proteins are encoded by the HRAS, KRAS and NRAS, genes and their protein products play a key role in such cellular processes as apoptosis, cell cycle, proliferation, differentiation, or migration of cells [20]. Because of its function, NF1 was classified in the group of suppressor genes (antioncogenes). Deficiency of the functional NF1 protein (NF1-/-) leads to a disturbance of cell homeostasis and lack of control over the Ras pathway, and consequently to uncontrolled proliferation. This in turn, may contribute to the initiation of the neoplastic transformation process. The catalytic activity of the NF1 depends on the central part of the protein, referred to as the GAP-related domain (GRD) [22].

The heterozygous mutation of the NF1 is responsible for approximately $95 \%$ of NF1 cases. In about half of the cases, the pathogenic mutation is inherited from one of the parents (in an autosomal dominant manner), while in the remaining $50 \%$ it is a de novo mutation [23]. The NF1 gene has the greatest mutation rates among human genes and to date, more than 2600 pathogenic variants thereof are known [23]. The NF1 mutations are characterised by complete penetration to adulthood and variable expression as a very wide spectrum of its expression is observed even in members of the same family (clinical symptoms, phenotype).

With a few exceptions, there are no data to show a correlation between the type of mutation and the clinical course of NF1 (genotype-phenotype correlation). The course of NF1 is extremely diverse, not only among members of the same family (having the same mutation), but even in individual patients at different times of their lives. There are some exception from the presented above:

- the group of patients with a large deletion that includes the NF1 gene and the adjacent regions. Their phenotype is more severe than in patients with mutations within the gene. Facial dysmorphic features, somatic hypertrophy, cognitive impairment, ADHD and the early appearance of a large number of cutaneous neurofibromas are observed [24],

- the group of patients with deletion of three base pairs in exon 17 (c.2970-2972 delAAT), which is associated with 
a milder disease course. Characteristic pigmentation features are observed, but cutaneous and plexus neurofibromas are absent [25],

- the group of patients with several variants of missense mutations contributing to substitution of other amino acids replacing arginine at codon 1809. These patients have café au lait spots, learning disabilities, shortness and lung stenosis, but no cutaneous neurofibromas and no clinically visible plexus neurofibromas [26].

There is also a form of NF1 that only affects certain parts of the body. This is called the segmental form. NF1 mutations are confined to the symptomatic areas of the body only. Therefore, there is no constitutional mutation in lymphocytes or fibroblasts, but only in cells from the segment affected by the disease. Most often, this form results from the appearance of the de novo mutation during embryogenesis (mosaicism) and usually does not affect germ cells (no risk of transmission of the disease). This disease is very difficult to diagnose [27].

\section{Genetic testing}

Diagnosis of NF1 is basically a clinical one, but genetic tests which allow identification of a mutation within the NF1 gene play an increasing role, especially for patients with suspected disease who do not meet the clinical criteria.

The recommended method is analysing the genome DNA (gDNA) sequence or only encoding sequence (cDNA) performed together with an analysis of rearrangements (microdeletion of individual exons) and/or deletion of the entire NF1 [28]. This approach identifies $95 \%$ of pathogenic NF1 variants in people meeting the NIH (US National Institutes of Health) diagnostic criteria. Pathogenic variants may occur throughout the whole NF1 gene (no hot spots). They vary, ranging from, among others, single nucleotide substitutions, small insertions to deletions (which may alter the reading frame or not), and also, approximately $22-30 \%$ of the mutations affect splicing. Therefore, the methods of cDNA analysis seem to be more valuable in terms of diagnostics [28, 29]. In cases of clinical suspicion of a microdeletion phenotype, identification of the NF1 gene deletion can be achieved using the following tests: FISH (with a specific probe), MLPA, qPCR or an array comparative genomic hybridisation test $(\mathrm{aCGH})$. The loss of the NF1 gene may be caused by chromosomal aberrations, which are detected in the cytogenetic test (most often translocations or inversions of chromosome 17 with the break point at q11.2). Chromosomal aberrations are responsible for NF1 in less than $1 \%$ of those affected by the disease [28].

Application of various algorithms allows identification of changes at a different level of effectiveness [28]:

- classic cytogenetic test - the aberration is detected in about $1 \%$ of patients,

- deletion analysis of the entire NF1 gene or its part - about $10 \%$,
- gDNA mutation analysis - 60-90\%,

- algorithm consisting of cDNA and gDNA analysis and rearrangement / deletion of the NF1 gene - above 95\%.

It is also worth mentioning that recent years have brought enormous development in genetic research and access to next-generation sequencing (NGS) platforms is increasingly easy, which allows for quick diagnostics. Currently, many companies offer gene panels for the analysis of various forms of phacomatoses, which means that several genes of key importance are sequenced simultaneously, e.g., NF1, NF2, SMARCB1, SPRED1, VHL, TSC1 or TSC2.

\section{Genetic counselling}

Identification of the pathogenic variant of the NF1 gene is extremely important for people in childbearing age, as it enables reproductive and family counselling - prenatal and preimplantation diagnostics are possible. Anyone carrying a NF1 mutation has a $50 \%$ risk of passing it on to their offspring. However, if a child from a previous pregnancy was diagnosed with NF1 mutation, but no such change was found in the parents, the risk of having another child with the disease is low [30]. On the other hand, germline mosaicism in one of the parents cannot be ruled out (mutation present only in germ cells). This involves significantly higher risk of another child's having the disease. Prenatal molecular tests can be performed on DNA isolated from trophoblast villi or amniocytes.

Genetic counselling is crucial for couples who decide to undergo prenatal NF1 testing due to the wide spectrum of symptoms and variable expression of the disease [30]. Life expectancy of patients with NF1 is about 8 years shorter than in the general population, especially due to the development of malignant neoplasms and vasculopathy [28].

\section{Type $\mathbf{2}$ neurofibromatosis}

Type 2 neurofibromatosis (MIM \# 101000) is an autosomal dominant disease of a frequency of 1:25,000-50,000 [31]. In $50 \%$ of cases NF2 mutation is inherited, and the other $50 \%$ are de novo mutations. The frequently observed mosaicism affects clinical symptoms of the disease.

Type 2 neurofibromatosis, like NF1, is a predisposing condition to neoplasms. The clinical picture is dominated by symptoms related to formation of schwannomas within the cranial, spinal and peripheral nerves, meningiomas, which may be located intracranially and intra-vertebrally, and ependymomas.

Bilateral auditory nerve vestibular neuroma (vestibular schwannoma) is pathognomonic for type 2 neurofibromatosis and occurs in $90 \%$ of patients [1]. Tinnitus, progressive retrocochlear hearing loss, dizziness and vertigo are the predominant symptoms in these patients. In the late stage of the disease, nausea and vomiting may occur. NF2 vestibular neuroma differs from neuromas that occur sporadically with polyclonal hyperplasia originating from distinct neoplastic cell lines that present a distinct type of NF2 mutation. This 
leads to lobularity identified in radiological examinations [32]. The increasing tumour mass causes progressive hearing loss, and if it enlarges significantly, it may compress the brain stem and trigger symptoms related to involvement of the facial nerves. Surgical treatment, which is the mainstay of the therapy, is technically difficult, and the number of relapses is more frequent in hereditary than in the sporadic form - 44\% vs. 1.3\% [1]. In the case of NF2-associated schwannoma, there is an increased risk of malignant transformation in response to radiotherapy, which limits the possibility of using this type of treatment [1]. There are attempts at using bevacizumab in chemotherapy, which is an inhibitor of vascular endothelial growth factor (VEGF) [33].

Schwannomas of other intracranial and peripheral nerves lead to paresis, e.g., facial muscles paresis (Bell's palsy), strabismus and sensory disturbances. In children, symptoms of the polio type with lower limb involvement can be observed [31]. Intracranial tumours, i.e., meningioma, glioma, ependymoma, may cause focal symptoms, convulsions, headaches, excessive drowsiness, vomiting with increasing intracranial pressure - mass effect. Meningiomas occur in approximately $50 \%$ of NF2 patients, largely in childhood, when the coexistence of meningiomas and NF2 is approximately 20\% [1]. Ependymomas, which are relatively rare compared to other types of neoplasms in NF2, are located mainly intramedullary in the cervical region and form characteristic corals (string of pearl) in the radiographic image [1].

Cranial neuromas and meningiomas are reported in about $50 \%$ of patients, neuromas of spinal nerves and peripheral trunks - In 40\% of patients [1]. While meningiomas significantly worsen the prognosis and are a common cause of death of patients with neurofibromatosis, ependymomas remain asymptomatic for a long time and are detected during periodic examinations in people with this disease.

Visual disturbances in patients with NF2 result from presence of optic nerve meningiomas, hamartoma-type tumours of the retinal pigment epithelium and posterior subcapsular cataract. On the other hand, skin lesions of neurofibroma character are less frequent than in NF1, and they characteristically grow hair. Subcutaneous neurofibromas, like café au lait spots are not numerous or may not occur at all.

\section{Diagnostic criteria for neurofibromatosis type 2 - Manchester criteria}

Leading criteria:

- bilateral tumour of the VIII nerve (shown in imaging - MR / CT or confirmed histologically),

- first degree relative with neurofibromatosis type 2 and unilateral tumour of the VIII nerve,

- first-degree relative with neurofibromatosis type 2, and finding two of the following: neurofibromas, meningiomas, schwannomas, gliomas, juvenile posterior subcapsular lens opacities.
Additional criteria:

- unilateral tumour of nerve VIII and any of the following: meningioma, glioblastoma, neurofibroma, schwannoma, posterior subcapsular lens opacities,

- multiple meningiomas (two or more) and unilateral tumour of the VIII nerve or any of the following: glioma, neurofibroma, schwannoma, cataract [1].

\section{Genetics of neurofibromatosis type 2}

The NF2 gene is located on the long arm of chromosome 22 (locus q12.2), encodes the protein called merlin (other names schwannomin, neurofibromin 2, MIM * 607379) composed of 595 amino acids weighing $70 \mathrm{kDa}$. The gene contains 17 exons, one of which is alternatively spliced. There are at least 8 different isoforms of this protein known [34]. Merlin is an acronym for moezin-ezrin-radixin-like protein, because this protein displays high homology with the cytoskeleton-related protein family 4.1. Merlin is involved in the formation of the submembrane cellular cytoskeleton, it connects actin fibres with the cell membrane or membrane glycoproteins. Like the NF1 gene, NF2 is a suppressor gene and its function is to inhibit cell proliferation (negative growth regulator), and to weaken adhesion and migrations, which are characteristic of neoplastic processes. Merlin is expressed in many tissues, especially in neurons and Schwann cells [35].

The genetic background of NF2 involves pathogenic alterations of the NF2 gene. NF2 is inherited as an autosomal dominant and fully penetrant disease. About $50 \%$ of people with NF2 have an affected parent, and the other 50\% develop the disease as a result of a de novo pathogenic variant. Mosaicism is frequently observed in NF2, affecting 30-60\% of cases with the de novo mutation. This means that only some of the patient's cells have the pathogenic lesion (mutated and wildtype alleles) and some have normal form of the gene (both wild-type alleles) [36]. Therefore, such a change may remain unidentified in standard genetic tests. The mosaic form is associated with a milder course of the disease and may be confined to certain areas of the body. In such a case, the risk of passing the change on to offspring is lower than $50 \%$. It depends on the number of reproductive cells with the pathogenic variant. However, if the change is passed on to the offspring, a more severe phenotype will be observed than that of the parent due to the fact that the child has mutations in all cells of the body [36-38]. A mosaic genotype can be detected by analysing the DNA from the tumour. If the same lesion is found in two tumours, the patient's offspring can then be tested for this genetic change [37]. As in NF1, after identifying the pathogenic variant of NF2 in the family, prenatal testing and genetic testing before implantation are possible.

Regarding the genotype-phenotype correlation, it was found that patients with NF2 caused by constitutional nonsense mutations (premature stop codon) or frame shift mutations (which lead to a shortening of the protein product) 
have a more severe disease compared to those with missense mutations (protein product of correct length) and to people with gene deletion (no protein product) [39]. Further, the differentiated phenotype is influenced by mutations at the splice sites. Patients with 5 'mutations have a more severe disease course compared to patients with mutations in 3 ' region. The type of mutation affects the relative risk of death, too. On the other hand, patients with missense mutations have a lower risk of death compared to patients with nonsense changes or frame shift [38].

\section{Genetic testing}

As in the case of NF1, a diagnostic approach to identifying the underlying NF2 lesion requires many steps. Depending on the phenotype, one can analyse the sequence of a single gene or a panel of several key genes, or use aCGH, exome sequencing, exome arrays or genome sequencing [40].

\section{Differential diagnosis}

When differentiating phacomatoses, it is necessary to take into account syndromes of phenotype similar to neurofibromatosis, including characteristic skin changes and a tendency to neoplastic growth

Legius syndrome is an autosomal dominant genetic disorder. It is characterised by skin pigmentation disorders with no accompanying features as in NF1. Difficulties in early diagnosis result from the similarity of both diseases and the varied expression of NF1 phenotypic traits in individuals. In Legius syndrome, skin manifestations include spots of the cafe au lait type (at least six), the number of which increases with age, and increased pigmentation of the armpits and groin. Macrocephaly, shortness, chest deformities, cognitive deficits, ADHD and retarded development may occur, too. However, neurofibromas, Lisch nodules and gliomas of the visual pathway - typical of NF1 are not found. Correct differentiation of Legius syndrome from neurofibromatosis 1 is extremely important due to differences in prognosis, which is significantly better in the case of Legius syndrome compared to NF1 $[40,41]$. The genetic background of Legius syndrome involves mutations in the SPRED1 gene located on the long arm of chromosome 15 (locus q13.2). Similarly as in NF1, the SPRED1 protein is a negative regulator of the RASMAPK pathway [41, 42].

Among other diseases that require differentiation from neurofibromatosis 1 and which include manifestation of cafe au lait spots, the following should be mentioned:

- constitutional mismatch repair deficiency (mismatch repair genes - MMR, MLH1, MSH2, MSH6, PMS1),

- multiple familial café au lait spots,

- syndromes:

- McCune-Albright (GNAS gene),

- Noonan (PTPN11, SOS1, RAF1, KRAS genes),

- Noonan with multiple lentigines (genes PTPN11, RAF1),
- multiple benign neoplasms, including hamartoma type tumours in syndromes:

- Proteus (AKT1 gene),

- Cowden (PTEN, KLLN, WWP1 genes)

- numerous orbital lesions of the neurofibroma type.

People with a suspicion of these diseases require genetic diagnosis, and if the diagnosis is confirmed, the entire family should obtain genetic counselling.

In Poland, from 15 June 2020, a pilot program is implemented in the field of coordinated medical care for patients with neurofibromatosis and related rasopathies based on the regulation of the Minister of Health. The objective of the programme is to improve effectiveness of diagnostics and treatment as well as early detection of health problems characteristic for this group of patients. Patients included in the program receive comprehensive care from a team of specialist physicians, including neurologists, psychiatrists, endocrinologists, otolaryngologists, surgeons, orthopaedists and others - depending on individual needs.

\section{Conflict of interest: none declared}

\section{Anna Kofla-Dłubacz}

Wroclaw Medical University

Faculty of Medicine

$2^{\text {nd }}$ Department of Paediatrics, Gastroenterology and Nutrition

ul. M. Curie-Skłodowskiej 50/52

55-369 Wrocław, Poland

e-mail:anna.kofla-dlubacz@umed.wroc.pl

Received: 19 May 2021

Accepted: 18 October 2021

\section{References}

1. Kresak JL, Walsh M. Neurofibromatosis: A Review of NF1, NF2, and Schwannomatosis. J Pediatr Genet. 2016; 5(2): 98-104, doi: 10.1055/s0036-1579766, indexed in Pubmed: 27617150.

2. Antônio JR, Goloni-Bertollo EM, Trídico LA. Neurofibromatosis: chronological history and current issues. An Bras Dermatol. 2013; 88(3): 329-343, doi: 10.1590/abd1806-4841.20132125, indexed in Pubmed: 23793209.

3. Neurofibromatosis. Archives of Neurology. 1988; 45(5): 575, doi: 10.1001/archneur.1988.00520290115023.

4. DeBella K, Szudek J, Friedman JM. Use of the national institutes of health criteria for diagnosis of neurofibromatosis 1 in children. Pediatrics. 2000; 105(3 Pt 1): 608-614, doi: 10.1542/peds.105.3.608, indexed in Pubmed: 10699117.

5. Korfhage J, Lombard DB. Malignant eriheral nerve sheath tumors: From eigenome to bedside. Mol Cancer Res. 2019; 17(7): 1417-1428, doi: 10.1158/1541-7786.MCR-19-0147, indexed in Pubmed: 31023785.

6. Seminog OO, Goldacre MJ. Risk of benign tumours of nervous system, and of malignant neoplasms, in people with neurofibromatosis: population-based record-linkage study. Br J Cancer. 2013; 108(1): 193-198, doi: 10.1038/bjc.2012.535, indexed in Pubmed: 23257896.

7. Park GH, Lee SJ, Yim H, et al. TAGLN expression is upregulated in NF1associated malignant peripheral nerve sheath tumors by hypomethylation in its promoter and subpromoter regions. Oncol Rep. 2014; 32(4): 1347-1354, doi: 10.3892/or.2014.3379, indexed in Pubmed: 25109740.

8. Katz D, Lazar A, Lev D. Malignant peripheral nerve sheath tumour (MPNST): the clinical implications of cellular signalling pathways. Expert Rev Mol Med. 2009; 11:e30, doi: 10.1017/S1462399409001227, indexed in Pubmed: 19835664. 
9. Evans DGR, Baser ME, McGaughran J, et al. Malignant peripheral nerve sheath tumours in neurofibromatosis 1. J Med Genet. 2002; 39(5): 311-314, doi: 10.1136/jmg.39.5.311, indexed in Pubmed: 12011145.

10. Watson KL, Al Sannaa GA, Kivlin CM, et al. Patterns of recurrence and survival in sporadic, neurofibromatosis Type 1-associated, and radiation-associated malignant peripheral nerve sheath tumors. J Neurosurg. 2017; 126(1): 319-329, doi: 10.3171/2015.12.JNS152443, indexed in Pubmed: 27035165.

11. Higham CS, Steinberg SM, Dombi E, et al. SARC006: Phase II Trial of Chemotherapy in Sporadic and Neurofibromatosis Type 1 Associated Chemotherapy-Naive Malignant Peripheral Nerve Sheath Tumors. Sarcoma. 2017; 2017: 8685638, doi: 10.1155/2017/8685638, indexed in Pubmed: 29138631.

12. Patwardhan PP, Surriga O, Beckman MJ, et al. Sustained inhibition of receptor tyrosine kinases and macrophage depletion by PLX3397 and rapamycin as a potential new approach for the treatment of MPNSTs. Clin Cancer Res. 2014; 20(12): 3146-3158, doi: 10.1158/1078-0432. CCR-13-2576, indexed in Pubmed: 24718867.

13. D'Adamo DR, Dickson MA, Keohan ML, et al. A Phase II Trial of Sorafenib and Dacarbazine for Leiomyosarcoma, Synovial Sarcoma, and Malignant Peripheral Nerve Sheath Tumors. Oncologist. 2019; 24(6): 857-863, doi: 10.1634/theoncologist.2018-0160, indexed in Pubmed: 30126857.

14. Hirbe A, Gutmann D. Neurofibromatosis type 1: a multidisciplinary approach to care. The Lancet Neurology. 2014; 13(8): 834-843, doi: 10.1016/s1474-4422(14)70063-8.

15. Bergqvist C, Servy A, Valeyrie-Allanore L, et al. NF France Network. Neurofibromatosis 1 French national guidelines based on an extensive literature review since 1966. Orphanet J Rare Dis. 2020; 15(1): 37, doi: 10.1186/s13023-020-1310-3, indexed in Pubmed: 32014052.

16. Agaimy A, Vassos N, Croner RS. Gastrointestinal manifestations of neurofibromatosis type 1 (Recklinghausen's disease): clinicopathological spectrum with pathogenetic considerations . Int J Clin Exp Pathol. 2012; 5(9): 852-862, indexed in Pubmed: 23119102

17. Liu S, Ran Li, Qi D, et al. Neovascular glaucoma in a pediatric patient with neurofibromatosis type 1: a case report. BMC Ophthalmol. 2020; 20(1): 168, doi: 10.1186/s12886-020-01438-5, indexed in Pubmed: 32345252.

18. Ferner RE, Huson SM, Thomas N, et al. Guidelines for the diagnosis and management of individuals with neurofibromatosis 1. J Med Genet. 2007; 44(2): 81-88, doi: 10.1136/jmg.2006.045906, indexed in Pubmed: 17105749.

19. Bergoug M, Doudeau M, Godin F, et al. Neurofibromin Structure, Functions and Regulation. Cells. 2020; 9(11), doi: 10.3390/cells9112365, indexed in Pubmed: 33121128.

20. Trovó-Marqui AB, Tajara EH. Neurofibromin: a general outlook. Clin Genet. 2006; 70(1): 1-13, doi: 10.1111/j.1399-0004.2006.00639.x, indexed in Pubmed: 16813595.

21. Abramowicz A, Gos M. Neurofibromin in neurofibromatosis type 1 mutations in NF1gene as a cause of disease. Dev Period Med. 2014; 18(3): 297-306, indexed in Pubmed: 25182393.

22. Scheffzek K, Welti S. Neurofibromin: Protein Domains and Functional Characteristics. Neurofibromatosis Type 1. 2012: 305-326, doi: 10.1007/978-3-642-32864-0 20.

23. Pacot L, Burin des Roziers C, Laurendeau I, et al. One Mutation may Conceal Another. Genes (Basel). 2019; 10(9), doi: 10.3390/genes10090633, indexed in Pubmed: 31443423.

24. Mautner VF, Kluwe L, Friedrich RE, et al. Clinical characterisation of 29 neurofibromatosis type-1 patients with molecularly ascertained $1.4 \mathrm{Mb}$ type-1 NF1 deletions. J Med Genet. 2010; 47(9):623-630, doi: 10.1136/ jmg.2009.075937, indexed in Pubmed: 20543202.

25. Upadhyaya M, Huson SM, Davies M, et al. An absence of cutaneous neurofibromas associated with a 3-bp inframe deletion in exon 17 of the NF1 gene (c.2970-2972 delAAT): evidence of a clinically significant NF1 genotype-phenotype correlation. Am J Hum Genet. 2007; 80(1): 140-151, doi: 10.1086/510781, indexed in Pubmed: 17160901.
26. Rojnueangnit K, Games A, Sharp A, et al. High Incidence of Noonan Syndrome Features Including Short Stature and Pulmonic Stenosis in Patients carrying NF1 Missense Mutations Affecting p.Arg1809: Genotype-Phenotype Correlation . Hum Mutat. 2015; 36(11): 1052-1063, doi: 10.1002/humu.22832, indexed in Pubmed: 26178382.

27. Freret ME, Anastasaki C, Gutmann DH. Independent mutations underlie café-au-lait macule development in a woman with segmental NF1. Neurol Genet. 2018; 4(4): e261, doi: 10.1212/NXG.0000000000000261, indexed in Pubmed: 30065955.

28. Friedman J. Neurofibromatosis 1 Synonyms: NF1, Von Recklinghausen Disease, Von Recklinghausen's Neurofibromatosis. In: Adam MP, Ardinger $\mathrm{HH}$, Pagon RA, et al. ed. GeneReviews ${ }^{\circledast}$. University of Washington, Seattle 1993-2021.

29. Morbidoni V, Baschiera E, Forzan M, et al. Hybrid Minigene Assay: An Efficient Tool to Characterize mRNA Splicing Profiles of Variants. Cancers (Basel). 2021; 13(5), doi: 10.3390/cancers13050999, indexed in Pubmed: 33673681.

30. Radtke HB, Sebold CD, Allison C, et al. Neurofibromatosis type 1 in genetic counseling practice: recommendations of the National Society of Genetic Counselors. J Genet Couns. 2007; 16(4):387-407, doi: 10.1007/ s10897-007-9101-8, indexed in Pubmed: 17636453.

31. Evans DG. Neurofibromatosis type 2 (NF2): a clinical and molecular review. Orphanet J Rare Dis. 2009; 4: 16, doi: 10.1186/1750-1172-4-16, indexed in Pubmed: 19545378.

32. Dewan R, Pemov A, Kim HJ, et al. Evidence of polyclonality in neurofibromatosis type 2-associated multilobulated vestibular schwannomas. Neuro Oncol. 2015; 17(4): 566-573, doi: 10.1093/neuonc/nou317, indexed in Pubmed: 25452392.

33. Plotkin SR, Duda DG, Muzikansky A, et al. Multicenter, Prospective, Phase II and Biomarker Study of High-Dose Bevacizumab as Induction Therapy in Patients With Neurofibromatosis Type 2 and Progressive Vestibular Schwannoma. J Clin Oncol. 2019; 37(35): 3446-3454, doi: 10.1200/JCO.19.01367, indexed in Pubmed: 31626572.

34. Chang LS, Akhmametyeva EM, Wu Y, et al. Multiple transcription initiation sites, alternative splicing, and differential polyadenylation contribute to the complexity of human neurofibromatosis 2 transcripts. Genomics. 2002; 79(1): 63-76, doi: 10.1006/geno.2001.6672, indexed in Pubmed: 11827459

35. Mindos T, Dun XP, North K, et al. Merlin controls the repair capacity of Schwann cells after injury by regulating Hippo/YAP activity. J Cell Biol. 2017; 216(2): 495-510, doi: 10.1083/jcb.201606052, indexed in Pubmed: 28137778.

36. Evans DG, Ramsden RT, Shenton A, et al. Mosaicism in neurofibromatosis type 2: an update of risk based on uni/bilaterality of vestibular schwannoma at presentation and sensitive mutation analysis including multiple ligation-dependent probe amplification. J Med Genet. 2007; 44(7): 424-428, doi: 10.1136/jmg.2006.047753, indexed in Pubmed: 17307835.

37. Evans DG, Sainio M, Baser ME. Neurofibromatosis type 2. J Med Genet. 2000; 37(12): 897-904, doi: 10.1136/jmg.37.12.897, indexed in Pubmed: 11106352.

38. Evans DGR, Baser ME, O'Reilly B, et al. Management of the patient and family with neurofibromatosis 2 : a consensus conference statement. Br J Neurosurg. 2005; 19(1): 5-12, doi: 10.1080/02688690500081206, indexed in Pubmed: 16147576.

39. Selvanathan SK, Shenton A, Ferner R, et al. Further genotype--phenotype correlations in neurofibromatosis 2. Clin Genet. 2010; 77(2): 163-170, doi: 10.1111/j.1399-0004.2009.01315.x, indexed in Pubmed: 19968670.

40. Evans DG. Neurofibromatosis 2. In: Adam MP, Ardinger HH, Pagon RA et al. ed. GeneReviews ${ }^{\oplus}$. University of Washington, Seattle 1993-2021.

41. Denayer E, Legius E. Legius Syndrome and its Relationship with Neurofibromatosis Type 1. Acta Dermato Venereologica. 2020; 100(7): adv00093-167, doi: 10.2340/00015555-3429.

42. Brems $\mathrm{H}, \mathrm{Chmara} M$, Sahbatou M, et al. Germline loss-of-function mutations in SPRED1 cause a neurofibromatosis 1-like phenotype. Nat Genet. 2007; 39(9): 1120-1126, doi: 10.1038/ng2113, indexed in Pubmed: 17704776. 